\title{
Tamaño de contenedor y tipo de sustrato afectan la eficiencia en el uso del agua en Gerbera jamesonii para flor cortada ${ }^{(1)}$
}

\author{
LIBERTAD MASCARINI(2); GABRIEL LORENZO(2); HECTOR SVARTZ(2); SABRINA PESENTI(2); SILVIAAMADO(2)
}

\begin{abstract}
RESUMEN
Se probaron dos tamaños de contenedor (macetas de 4 y 8 L) y tres mezclas de sustrato (\% de turba/perlita $0 / 100,25 / 75$ y 50/50) con el objetivo de determinar la combinación óptima para el cultivo de gerbera para flor cortada, considerando la productividad comercial (cantidad y calidad de flores) y el impacto ambiental (eficiencia en el uso del agua y fertilizantes). La mejor combinación en el primer año de producción fue macetas de $8 \mathrm{~L}$ con sustrato $0 / 100$, seguido de los contenedores de 4 y 8 L con $25 / 75$, con un rendimiento de 53.4 , 30.5 y 29.7 flores. $/ \mathrm{m}-2$ respectivamente. El resto de los tratamientos tuvo un rendimiento similar de alrededor de 20 flores $\mathrm{m}-2$. La calidad comercial de las flores cortadas fue aceptable en todos los tratamientos. En cuanto a la eficiencia medida como agua consumida por flor cosechada, el tratamiento 8 L 0/100 resultó claramente superior con un valor de $1.23 \mathrm{~L} /$ flor, el resto de los tratamientos estuvieron entre 2.17 y $4.14 \mathrm{~L} /$ flor, guardando relación tanto con el tamaño de contenedor como con el tipo de sustrato utilizado. La importancia de estos resultados es la consideración simultánea de los factores que hacen a la rentabilidad comercial y al desarrollo de tecnologías sustentables para los cultivos intensivos.
\end{abstract}

Palabras-chave: agricultura sustentable, cultivo sin suelo, perlita, turba.

\section{ABSTRACT \\ Container size and growing media affects water use efficiency in a Gerbera jamesonii cut flower crop}

Two containers (4 and $8 \mathrm{~L}$ plastic pots) and three growing media mixture types (0/100, 25/75 and 50/50\% of peat/perlite) were used, with the aim of determining the optimum combination for gerbera cut flower crop, considering both commercial productivity (yield and quality of cut flowers) and environmental impact, (water and fertilizer use efficiency). The best combination in the first year of production was $8 \mathrm{~L}$ and $0 / 100$, followed of 4 and $8 \mathrm{~L}$ with the 25/75, with a yield of 53.4, 30.5 and 29.7 flowers $\mathrm{m}-2$ respectively. The yield of the rest of the treatments were similar, around 20 flowers $\mathrm{m}-2$. Commercial cut flower quality was aceptable in all treatments. Considering the efficiency measured as the ratio water used per flower harvested, 8L 0/100 was clearly superior with a value of $1.23 \mathrm{~L} /$ flower, the rest of the treatments were between 2.17 y 4.14 L/flower, keeping relation as much with container size like with growing media mixture. The importance of these results is the simultaneous consideration of many factors which affect both comercial profitability and development of sustainable technology for intensive crops.

Keywords: sustainable agriculture, soilless culture, perlite, peat.

\section{INTRODUCCIÓN}

El agua es un recurso natural imprescindible que limita cada vez más las actividades económicas que hacen uso intensivo de ella, como la agricultura y la ganadería, compitiendo además con el consumo humano. Atendiendo a las previsiones de cambio climático global, estas limitaciones se profundizarán enormemente en los próximos años (MEDRANO et al., 2007). La industria de plantas ornamentales se verá afectada por la disminución de recursos hídricos para riego en el mediano plazo ya sea por una restricción de la oferta como por un aumento del costo del agua, por lo tanto, es obligatorio mejorar los sistemas productivos en orden de lograr una mayor eficiencia (BEESON Jr. et al., 2004). Se prevé que la intensificación sostenible de la producción puede ser una respuesta al cambio climático en algunos agroecosistemas intervenidos. Una estrategia de intensificación de la producción es el uso del riego y las tecnologías que permitan un uso eficiente del agua y el suelo. También se encuentran aquellas estrategias de producción que promueven un uso más eficiente de insumos (incluye al riego) a través de especies mejoradas que logren una mayor producción de masa seca por volumen de agua, o aquellas que logran una mayor respuesta a la aplicación de fertilizantes. El uso eficiente del agua y fertilizantes resultará, por un lado, en un menor impacto ambiental de la producción $y$, por otro, en un mayor beneficio económico.

La producción ornamental es uno de los rubros más dinámicos de la economía mundial, con una tasa de crecimiento de alrededor del $6 \%$, y gran generador de empleos (CARBONE, 2007). Se realiza bajo la modalidad de agricultura intensiva y además de concentrar una gran demanda de agua en una pequeña superficie, puede ser potencialmente contaminante debido al uso de fertilizantes (TILMAN et al., 2002; OLYMPIOS, 2005). Sumado a ello, la mayoría de los centros productores de plantas ornamentales se localizan en la periferia de los grandes centros urbanos; así, por ej., el grueso de la producción ornamental de Argentina se concentra en un radio de 150 $\mathrm{km}$ alrededor de la ciudad de Buenos Aires (GARCÍA FILGUERA y DI STÉFANO, 2002). La técnica de cultivo

\footnotetext{
(1) Recebido em 04/02/2010 e aceito para publicação em 09/08/2011.

(2) Facultad de Agronomía - Universidad de Buenos Aires. Avenida San Martín 4453 (1417) Ciudad Autónoma de Buenos Aires, Argentina
} 
sin suelo es usada comercialmente para la producción de plantas ornamentales de alto valor (CALATAYUD et al., 2008). La misma, respecto a la de cultivo en suelo, ofrece varias ventajas al productor, como ser el control más estricto de la cantidad de agua y fertilizantes aplicados (JENSEN, 1997), reducción de problemas sanitarios (RIVIERE y CARON, 2001), y un aumento de la productividad de 3 a 10 veces, junto con un menor impacto ambiental debido a un menor uso de agroquímicos como el bromuro de metilo (CANTLIFFE y VANSICKLE, 2001). El uso de sustratos, en dicha técnica, requiere un adecuado balance entre aireación y disponibilidad de agua para lograr un desarrollo normal del cultivo (FAKHRI et al., 1995). Por tanto, es necesario probar diferentes sustratos para alcanzar una porosidad adecuada, combinando las propiedades hidráulicas y las características físicas de cada material o derivado de la mezcla de dos o más materiales (VALENZUELA et al., 2004).

En la producción intensiva ornamental, Gerbera jamesonii Bolus se cultiva para flor cortada o planta en maceta, con una vida útil de cinco años aunque se recomienda reemplazarla a los tres años (BAÑóN-ARIAS et al., 1993). Como flor cortada, ocupa el $5^{\circ}$ lugar en ventas en la subasta holandesa, alcanzando la cifra 117 millones de euros en el año 2008 Vereniging van Bloemenveilingen in Netherland (VBN, 2009). Se adapta al sistema de cultivo sin suelo, pero es necesario el ajuste de esta innovación a cada situación particular para mejorar la eficiencia física y la calidad de los productos, y así alcanzar mejores oportunidades de comercialización(GRAFIADELLIS et al., 2000). La evaluación económica de esta producción deberá considerar tanto los costos (volumen de sustrato, tipo de contenedor, volumen de agua y fertilizante aplicado) como los ingresos (cantidad y calidad de flores producidas). Este trabajo parte de la hipótesis que existe una combinación sustrato-contenedor que logrará optimizar la productividad con el menor impacto ambiental y que resultará en un mayor beneficio económico.

Los objetivos de este trabajo fueron: 1) cuantificar la eficiencia en el uso del agua en un cultivo de gerbera para flor de corte en diferentes tamaños de contenedor y sustratos y 2) verificar si diferentes contenedores y mezcla de sustratos influyen sobre la cantidad y la calidad de flores, en el primer año de producción.

\section{MATERIALES Y MÉTODOS}

El experimento se efectuó en la Facultad de Agronomía, Universidad de Buenos Aires (34.55 $\mathrm{S}, 58.29^{\circ} \mathrm{O} ; 25 \mathrm{~m}$ de altitud). La temperatura media del mes más frío es $10.3{ }^{\circ} \mathrm{C}$ (julio) y la del mes más cálido $23.4{ }^{\circ} \mathrm{C}$ (enero). La radiación global mensual es $7.03 \mathrm{MJ} \mathrm{m}^{-2}$ (julio) y $22.98 \mathrm{MJ} \mathrm{m}^{-2}$ (enero). La evapotranspiración potencial del cultivo de gerbera en invernadero, calculada por el método de Penman-Monteith, es $2.95 \mathrm{~mm} \mathrm{~d}^{-1}$ (julio) y $6 \mathrm{~mm} \mathrm{~d}^{-1}$ (enero) (MASCARINI et al., 2001).

Se cultivaron plantas de Gerbera jamesonii cv "Sangría" obtenidas por cultivo in vitro (procedencia Florist ${ }^{\circledR}$, Holanda), en macetas plásticas cilíndricas individuales de 8 y $4 \mathrm{~L}$ a una densidad de 6.1 plantas $\mathrm{m}^{-2}$, realizando la plantación en enero de 2006. Los contenedores fueron de igual diámetro, aumentando su altura en función del volumen, ubicándose los mismos sobre una mesada de 60 $\mathrm{cm}$ de altura. Los sustratos fueron preparados con diferentes proporciones de turba y perlita (Tabla 1).

El cultivo se ubicó dentro de un invernadero metálico con una cubierta de polietileno de 150 micrones, con orientación NNO-SSE. La ventilación fue mediante la apertura automática de las ventanas laterales, cuando la temperatura interna alcanzaba $23{ }^{\circ} \mathrm{C}$. La calefacción se realizó con un generador de aire caliente con termostato, el que encendía cuando la temperatura interior descendía de $\operatorname{los} 15^{\circ} \mathrm{C}$. El fertirriego fue aplicado automáticamente mediante goteros tipo espagueti $\left(\right.$ Netafim $\left.^{\circledR}\right)$ con un caudal de $2 \mathrm{~L} \mathrm{~h}^{-1}$. La cantidad de agua se calculó en función de la evapotranspiración del cultivo (MASCARINI et al., 2001). La solución nutritiva normal tuvo la siguiente composición (en mmol L-1): $9.95 \mathrm{NO}_{3}^{-}, 0.5 \mathrm{H}_{2} \mathrm{PO}_{4}^{-}, 6 \mathrm{~K}^{+}, 1.8 \mathrm{Ca}^{2+}, 0.45$ $\mathrm{Mg}^{2+}$ y micronutrientes. La relación $\mathrm{N}: \mathrm{P}_{2} \mathrm{O}_{5}: \mathrm{K}_{2} \mathrm{O}$ fue 1 : $0.14: 2$. El pH y CE de la solución de riego se mantuvieron entre 6 - 6.5 y 1.5 - $1.8 \mathrm{dS} \mathrm{m}{ }^{-1}$, respectivamente.

La cosecha se realizó cuando dos círculos de flores liguladas de la inflorescencia mostraban granos de polen. Se determinó número de flores cosechadas, longitud del pedúnculo, diámetro del capítulo y diámetro del pedúnculo a $10 \mathrm{~cm}$ por debajo de la inflorescencia, durante el primer año de cultivo. El agua drenada de cada maceta se recolectó en bidones plásticos de $10 \mathrm{~L}$ debajo de la mesada, la cual estaba cubierta con un polietileno negro, así como también se recolectó el agua aplicada mediante tres bidones que recogían directamente el agua de los goteros. En cada uno de estos bidones se midió el volumen, el $\mathrm{pH}$ y la $\mathrm{CE}$ del agua drenada y de la solución nutritiva dos veces a la semana con un peachímetro-conductímetro (Hanna Instruments $\left.{ }^{\circledR}\right)$. El consumo de agua se calculó como la diferencia de volumen entre riego aplicado - agua drenada, incluyendo este consumo tanto transpiración por el cultivo como evaporación desde el sustrato. Sin embargo, durante gran parte del ensayo, las hojas de las plantas cubrían totalmente la superficie del contenedor, por lo tanto se consideró despreciable la evaporación de agua desde el sustrato. Con los datos de consumo de agua y producción total se determinó la eficiencia en el uso del agua en términos de cantidad de flores producidas por L de agua utilizado por el cultivo.

El diseño experimental fue al completamente aleatorizado con arreglo factorial de tratamientos, con dos factores: volumen de contenedor (dos niveles) y mezcla de sustratos (tres niveles). La unidad muestral fue la planta, cada tratamiento tuvo 10 repeticiones. Los datos se analizaron por ANOVA y contrastes ortogonales y se usó la prueba de Tukey $(\mathrm{p} \leq 0.05)$ para comparar las medias de los tratamientos, con el software INFOSTAT v1.1 (INFOSTAT/ PROFESIONAL, 2002)

\section{RESULTADOS}

El período del experimento fue dividido en dos ciclos de crecimiento: ciclo 1, otoño-invierno, desde marzo hasta 
julio y ciclo 2, primavera, desde agosto hasta noviembre,

En la tabla 2 se presentan los resultados obtenidos en el ciclo 1. La mejor calidad de flor se obtuvo en los tratamientos $8 \mathrm{~L} 1$ y $8 \mathrm{~L} 2$, que mostraron una mayor longitud de pedúnculo y mayor diámetro de capítulo, mientras que el tratamiento 8L3 y 4L1 fueron inferiores en cuanto a diámetro del capítulo. El tratamiento 8L3 fue el más productivo, con 18.3 flores $\mathrm{m}^{-2}$, mientras que no se observaron diferencias significativas entre los restantes tratamientos. El consumo de agua fue similar en todos los tratamientos, siendo 4L1 el más bajo y 8L1 el más alto. Las diferencias de longitud fueron atribuidas al tamaño de contenedor, al tipo de sustrato y a la interacción entre contenedor y sustrato; mientras que las diferencias de diámetro solo fueron atribuibles a la interacción entre los dos factores. Por otro lado las diferencias en producción $\mathrm{y}$ en consumo de agua se relacionaron con el tamaño de contenedor y con la interacción (Tabla 3).

En la tabla 2B se presentan los resultados obtenidos en el ciclo 2 . La calidad fue muy pareja en todos los tratamientos, a excepción de 4L1 que mostró una longitud menor y de 8L3 cuyo diámetro de pedúnculo fue ligeramente menor. El tratamiento 8L3 fue el más productivo pero 8L2, 4L3 y 4L2, es decir, los que tenían mayor proporción de perlita en el sustrato fueron significativamente similares $(35.4,21.4$, 16.8 y 21.4 flores $\mathrm{m}^{-2}$ respectivamente). El consumo de agua fue significativamente mayor en los contenedores de $8 \mathrm{~L}$, no encontrándose diferencias entre distintos sustratos. Este efecto fue atribuido al tamaño de contenedor, tipo de sustrato y a la interacción entre ellos (Tabla 3).

Considerando el período completo, es decir, sumando ambos ciclos, 8L 3 fue claramente el más productivo con 58.4 flores $\mathrm{m}^{-2}$, el resto de tratamientos alcanzó valores entre 23.1 y 40.2 flores $\mathrm{m}^{-2}$ (8L1 y $4 \mathrm{~L} 3$ respectivamente), se observó que la mayor eficiencia en el uso del agua se obtuvo con el contenedor de $8 \mathrm{~L}$ y con sustrato $100 \%$ perlita (Figura 1) y que ésta disminuía a medida que se agregaba turba a la mezcla en los contenedores de 8 L. Esto fue atribuido a la menor producción en los tratamientos con turba, ya que el consumo absoluto de agua fue similar. En cuanto a los contenedores de $4 \mathrm{~L}$ el consumo de agua absoluto también fue similar, pero la inclusión de un $25 \%$ de turba contribuyó a mejorar la eficiencia por la mayor productividad. El mismo comportamiento se observa si se consideran ambos ciclos por separado (datos no mostrados), con la única diferencia que en el ciclo 2 el sustrato 50/50 presenta una eficiencia similar independientemente del tamaño de contenedor, atribuible a un considerable aumento en la productividad en 4L1 al mismo tiempo que desciende en 8L1 (Tabla 2B).

\section{DISCUSIÓN}

Durante el primer año del cultivo de gerbera, la mayor producción de flores fue obtenida con $100 \%$ perlita en contenedores de $8 \mathrm{~L}$, habiendo diferencias significativas con el resto de los tratamientos desde estadios muy tempranos, lo cual podría estar mostrando el efecto de la restricción al crecimiento de las raíces impuesta por contenedores más pequeños y el de la incorporación de turba a la mezcla de sustratos. Esto concuerda con estudios anteriores que reportaron una reducción del crecimiento vegetativo de la parte aérea cuando fue restringido el crecimiento de la raíz, por ejemplo, en Citrus sp. (MATAA y TOMINAGA, 1998), Prunus sp. (BOLAND et al., 2000), Salvia sp. (VAN IERSEL, 1997) y Lycopersicon sp. (NISHIZAWA y SAITO, 1998).

El consumo de agua medido a través de la diferencia riego-drenaje fue significativamente mayor en los contenedores de $8 \mathrm{~L}$ para todos los sustratos. Las diferencias encontradas en este ensayo pueden atribuirse tanto al contenedor como al sustrato. La capacidad de contenedor (CC) se define como porcentaje volumétrico de agua que es retenido por el sustrato en un contenedor de altura h, una vez saturado y dejando drenar libremente sin sufrir evaporación (WHITE y MASTALERZ, 1966). Para un determinado sustrato la CC será menor cuanto mayor sea $\mathrm{h}, \mathrm{y}$ además habrá una zona saturada en la base del mismo, independiente de las características del recipiente (SPOMER, 1974; BUNT, 1976). En nuestro caso los contenedores de 4 y $8 \mathrm{~L}$ difieren en la altura por lo tanto en la capacidad de contenedor, pero a la vez los contenedores de $8 \mathrm{~L}$ tienen el doble de volumen, por lo que la cantidad absoluta de agua aplicada para saturar el espacio poroso fue mayor. Por lo tanto, el volumen de drenaje del agua gravitacional hasta alcanzar la CC fue proporcionalmente mayor en contenedores de $4 \mathrm{~L}$, es decir mayor porcentaje de agua drenada por unidad de agua aplicada. La altura del contenedor a su vez determina la distancia mínima desde la planta hasta la zona de saturación permanente, es decir, en los contenedores de $8 \mathrm{~L}$ hay mayor proporción de sustrato no saturado en relación al volumen total, por lo tanto mejores condiciones para las raíces.

El exceso de agua en la rizosfera determina menor disponibilidad de oxígeno, lo cual podría afectar el crecimiento de las raíces y la absorción de nutrientes (BAAS et al., 2001). Además, un drenaje más intenso podría contribuir a una más rápida estabilización de las condiciones químicas del sustrato, lixiviando iones indeseables fuera del sistema.

De acuerdo con los resultados de VENCE y MARTINEZ (2008a y 2008b), las diferencias encontradas entre sustratos pueden explicarse a partir de las características físicas de los mismos, relacionadas con la capacidad de aireación (CA), el porcentaje de agua fácilmente disponible (AFD) y la capacidad de retención de agua (CRA), mientras que las diferencias entre contenedores podrían atribuirse a la diferente capacidad de contenedor derivada de la altura y el volumen total. Analizando los datos de producción de los contenedores de $8 \mathrm{~L}$, se observa que a medida que se incluyó turba en la mezcla, la cantidad de flores cosechadas disminuyó, probablemente debido a una menor capacidad de aireación lo que determinó condiciones de crecimiento subóptimas. En el caso de los contenedores de $4 \mathrm{~L}$ y diferentes sustratos, los resultados de este ensayo indicarían que la situación óptima se obtiene con un $25 \%$ de turba y $75 \%$ perlita. Con perlita $100 \%$ en contenedores pequeños, a pesar de la mayor CC el menor volumen total sumado al menor AFD, limitaría la disponibilidad de agua para la planta, mientras que el agregado de turba permitiría una mayor retención entre riegos, debido a una mayor CRA 
y mayor AFD, aunque altas proporciones afectarían la CA, lo cual podría explicar la disminución de la producción en $4 \mathrm{~L} 1$.

Todos los tratamientos lograron índices de calidad aceptables para la producción comercial, ya que las diferencias numéricas encontradas no representan en la práctica diferencias de calidad apreciables. En términos generales se podría decir que, en el primer año de producción, se obtuvieron mejores resultados en cuanto a longitud de pedúnculos en los contenedores de $8 \mathrm{~L}$, mientras que los de $4 \mathrm{~L}$ fueron más convenientes para lograr capítulos de mayor diámetro. LORENZO (2003) reportó que cuando se obtuvieron flores más largas, sus capítulos fueron más pequeños, aparentemente debido a una partición diferencial de fotoasimilados, lo cual se relacionaría con el grado de restricción a las raíces. XU y KAFKAFI (2001) encontraron que la restricción a las raíces durante los primeros estadios de pimiento resultó en mayor precocidad y en un incremento de la distribución de fotoasimilados hacia los órganos reproductivos. Efectos similares se describieron para algodón (BENPORATH y BAKER, 1990) y tomate (PETERSON et al., 1991). En plantines de Pinus pinea, el volumen del contenedor tuvo gran influencia en la morfología de la planta. Los de mayor volumen tuvieron plantas de mayor altura, diámetro y volumen de nutrientes, y la actuación en campo fue buena (DOMÍNGUEZ-LERENA et al., 2006). En contenedores de mayor volumen la mezcla de sustratos produce menores variaciones de calidad de flor respecto a los de menor volumen. Así, el contenedor de 4 L con más de $50 \%$ de perlita produjo mayor diámetro de capítulo, largo y diámetro de tallo, lo que concuerda con las investigaciones de HEISKANEN y RIKALA (2003). La restricción a las raíces impuesta por el contenedor y por el tipo de sustrato podría explicar la reducción de la calidad a largo plazo cuando se utilizan altos porcentajes de turba. Para Prunus avium L. se ha reportado que aunque la restricción a las raíces incrementó el número de flores, también comprometió fuertemente la cantidad de frutos (WHITE et al., 2001).

En los cultivos hidropónicos o sin suelo, el agua es además fuente de nutrientes para la planta, de manera que una mayor eficiencia en el uso del agua también lo será de fertilizantes. Este punto es especialmente sensible dado la potencial fuente de contaminación que representan los cultivos intensivos como lo son los cultivos para flor de corte. La regulación legal del uso del agua es un ejemplo de la dimensión del problema, que se espera se haga más estricta en el mediano plazo, no solamente respecto a la restricción de la cantidad de agua disponible, sino también en cuanto al volumen de fertilizantes aplicados y aún la concentración de los mismos en los efluentes (BEESON JR. et al., 2004). En ese sentido, es importante recalcar el impacto positivo en los agroecosistemas derivado de la intensificación sostenible donde el uso de los recursos ambientales renovables garantizan tanto la calidad de vida de los propios agricultores como la conservación de los mismos a fin de sostener la estructura productiva.

En condiciones de cultivo comercial, y en orden de optimizar el beneficio económico en armonía con la sustentabilidad del sistema, se debería apuntar a incrementar los rendimientos físicos haciendo además un uso más eficiente de los recursos. TILMAN et al. (2002) afirman que el $40 \%$ de la producción agrícola proviene del $16 \%$ de la superficie cultivable, la cual corresponde a las tierras irrigadas. Sin embargo, el ritmo de crecimiento de la población mundial y por lo tanto de la demanda por alimentos, sumado a que el consumo humano cada vez más compite por el agua con las actividades agrícolas, obliga a aumentar la eficiencia en forma sostenida.

\section{CONCLUSIONES}

El aumento de la eficiencia en el uso del agua posibilitaría por un lado producir en forma más amigable con el medio ambiente y, por otro, manejar racionalmente un recurso escaso. La combinación contenedor-sustrato 8 L $100 \%$ perlita es, entre las ensayadas en este trabajo, la indicada para optimizar la cantidad y calidad de flores y el uso del agua y nutrientes durante el primer año de producción, lo cual resultará en una mayor rentabilidad comercial con un bajo impacto ambiental.

\section{REFERENCIAS}

BAAS, R., WEVER, G., KOOLEN, A.J., TARIKU, E., STOL, K.J. Oxygen supply and consumption in soilles culture: evaluation of fan oxygen simulation model for cucumber. Acta Horticulturae, v.554, p.157-161, 2001.

BAÑÓN ARIAS, S., CIFUENTES ROMO D., FERNÁNDEZ HERNANDEZ J.A., GONZALEZ BENAVENTE A. Gerbera, lilium, tulipán y rosa. Madrid, España: Mundiprensa, 1993. 250 p.

BEESON Jr., R.C., ARNOLD, M.A., BILDERBACK, T.E., BOLUSKY, B., CHANDLER, S., GRAMLING, H.M., LEA-COX, J.D., HARRIS, J.R., KLINGER, P.J., MATHERS, H.M., RUTER, J.M., YEAGER, T.H. Strategic vision of container nursery irrigation in the next ten years. Journal of Environmental Horticulture, v.22, n.2, p.113-115, 2004.

BEN-PORATH, A., BAKER, D.N. Taproot restriction effects on growth, earliness, and dry weight partitioning of cotton. Crop Science, v.30, p.809-814, 1990.

BOLAND, A.M., JERIE, P.H., MITCHELL, P.D., GOODWIN, I., CONNOR, D.J. Long-term effects of restricted root volume and regulated deficit irrigation on peach: II. Productivity and water use. Journal of the American Society of Horticultural Science, v.125, p.143-148, 2000.

BUNT, A.C. Modern Potting Composts: A manual on the preparation and use of growing media for pot Plants. Pennsylvania, USA: University Park, PA: The Pennsylvania State University Press, 1976. 277 p.

CAlATAYUD, A., GORBE, E., ROCA, D., MARTÍNEZ, 
P.F. Effect of two nutrient solution temperatures on nitrate uptake, nitrate reductase activity, NH4+ concentration and chlorophyll a fluorescence in rose plants. Environmental and Experimental Botany, v.64, p.65-74, 2008.

CANTLIFFE, D.J., VANSICKLE, J.J. Competitiveness of the Spanish and dutch greenhouse industries with the Florida fresh vegetable industry. Proceedings of the Florida State Horticultural Society, v.114, p.283-287, 2001.

CARBONE, F. Brotes de exportación. Diario La Nación. Comercio Exterior. Artículo publicado. Disponível em: http://www.lanacion.com.ar/nota.asp?nota_id=882539. Acesso em: 13 de Febrero de 2007.

DOMÍNGUEZ-LERENA, S., HERRERO, S.N., CARRASCO, M.I., OCAÑA, B.L., PEÑUELAS, R.J.L., MEXAL, J.G. Container characteristics influence Pinus pinea seedling development in the nursery and field. Forest Ecology and Management, v.221, n.1-3, p.63-71, 2006.

FAKHRI, M.N., MALOUPA, E., GERASOPOULOS, D. Effects of substrate and frequency of irrigation on yield and quality of three Gerbera jamesonii cultivars. Acta Horticulturae, v.407, p.41-45, 1995.

GARCÍA FILGUEIRA, S., DI STÉFANO, D. Informe de Situación del Sector Floricultura en Argentina. Disponível em: http://www.cfired.org.ar/Default.aspx?nId=4070. 2002

GRAFIADELLIS, I., MATTAS, J., MALOUPA, E., TZOURAMANI, I., GALANOPOULOS, K. An economic analysis of soilless culture in gerbera production. HortScience, v.35, n.2, p.300-303, 2000.

HEISKANEN, J., RIKALA, R Effect of peat-based container media on establishment of Scots Pine, Norway Spruce, and Silver Birch seedlings. Tree Planters' Notes, v.50, n.1, p.28-33, 2003.

INFOSTAT/PROFESIONAL. Versión 1.1. Universidad Nacional de Córdoba. Estadística y Biometría. Facultad de Ciencias Agrarias, Córdoba, Argentina. Disponível em: http://www.infostat.com.ar. Acesso em:15 Aug. 2002.

JENSEN, M.H. Hydroponics. Hortscience, v.32, p.10181021, 1997.

LORENZO, G.A. Rendimiento y calidad de dos variedades de Gerbera jamesonii y su relación con el tamaño de contenedor en el primer año de producción. In: Floricultura en la Argentina: Investigación y tecnología de producción. MASCARINI, L., VILLELA, F., WRIGHT, E. (Eds.). Editorial Facultad de Agronomía, Universidad de Buenos Aires, Argentina, 2003. p. 253-261.

MASCARINI, L., DELFINO, O.S., VILELLA, F. Evapotranspiration of two Gerbera jamesonii cultivars in hydroponics: adjustment of models for greenhouses. Acta Horticulturae, v.554, p.261-269, 2001.
MATAA, M., TOMINAGA, S. Effects of root restriction on tree development in Ponkan mandarin. Journal of the American Society of Horticultural Science, v.123, p.651-655, 1998.

MEDRANO, H., BOTA, J., CIFRE, J., FLEXAS, J., RIBAS-CARBÓ, M., GULÍAS, J. Eficiencia en el uso del agua por las plantas. Investigaciones geográficas, v.43, p.63-84, 2007.

NISHIZAWA, T., SAITO, K. Effects of rooting volume restriction on the growth and carbohydrate concentration in tomato plants. Journal of the American Society of Horticultural Science, v.123, p.581-585, 1998.

OLYMPIOS, C.M. Overview of soilless cultura: advantages, constraints and perspectivas for its use in mediterranean countries. Cahiers Options Mediterranéennes, v.31, p.307-324, 2005.

PETERSON, T.A., REINSEL, M.D., KRIZEK, D.T. Tomato (Lycopersicon esculentum Mill., cv.'Better Bush') plant response to root restriction. Journal of Experimental Botany, v.42, n.243, p.1233-1240, 1991.

RIVIERE, L.M., CARON, J. Research on substrates: state of the art and need for the coming 10 years. Acta Horticulturae, v.548, p.29-41, 2001.

SPOMER, L.A. Two classroom exercises demonstrating the pattern of container soil water distribution. HortScience, v.9, n.2, p.152-153, 1974.

TILMAN, D., CASSMAN, K.G., MATSON, P.A., NAYLOR, R., POLASKY, S. Agricultural sustainability and intensive production practices. Nature, v.418, p.671677, 2002.

VALENZUELA, O.R., GALLARDO, C.S., RODE ,M.I. Caracterización de algunos materiales clásicos utilizados en la formulación de sustratos. In: Congreso Argentino de Floricultura y Plantas Ornamentales, 2, Buenos Aires, Argentina, 2004. Resumen... Buenos Aires, Argentina, INTA, 2004. p.200-202.

VAN IERSEL, M.W. Root restriction effects on growth and development of salvia (Salvia splendens). HortScience, v.32, p.1186-1190, 1997.

VBN (Vereniging van Bloemenveilingen in Nederland). Horticultura ornamental holandesa. Leiden, Holanda: Ed. Oficina Holandesa de Flores. 2009. Disponível em: www.flowercouncil.org.

VENCE, L., MARTINEZ, D. Caracterización física de 4 sustratos para plantas. Parte I: determinación de la densidad de sustrato seca, capacidad de retención de agua, porosidad total y porosidad de aire por 2 métodos. In: Congreso Argentino de Floricultura y plantas ornamentales, 4, Jornadas nacionales de Floricultura, 10, Corrientes, Argentina. Resumen... Corrientes, Argentina, 2008. p. 
$236-240$

VENCE, L., MARTINEZ, D. Caracterización física de 4 sustratos para plantas. PARTE II: curvas de liberación de agua obtenidas con dos dispositivos de tensión diferentes. In: Congreso Argentino de Floricultura y plantas ornamentales, 4, Jornadas nacionales de Floricultura, 10, Corrientes, Argentina. Resumen... Corrientes, Argentina, 2008. p. $241-246$.

WHITE, M.D., TUSTIN, D.S., FOOTE, K.F., CAMPBELL, J.M. Growth of young sweet cherry trees in response to root restriction using root control bags. Acta Horticulturae, v.557, p.391-398, 2001.

WHITE, J.W., MASTALERZ, J.W. Soil moisture as related to "container capacity". Journal of the American Society of Horticultural Science, 89: 758-765, 1966.

XU, G., KAFKAFI, U. Nutrient supply and container size effects on flowering, fruiting, assimilate allocation and water relations of sweet pepper. Acta Horticulturae, v.554, p.113-120, 2001.

Tabla 1. Composición del sustrato (porcentaje de turba y de perlita en la mezcla), y volumen de contenedores (4 y 8 L) utilizados en el ensayo

Table 1. Growing media composition (percentage of peat and perlite in the mixture), and container size (4 and $8 \mathrm{~L})$ used in the experiment

\begin{tabular}{cccc}
\hline \multicolumn{2}{c}{ Mezcla de sustrato } & \multicolumn{2}{c}{ Volumen de contenedor } \\
Turba (\%) & Perlita (\%) & $4 \mathrm{~L}$ & $8 \mathrm{~L}$ \\
\hline 50 & 50 & $4 \mathrm{~L} 1$ & $8 \mathrm{~L} 1$ \\
25 & 75 & $4 \mathrm{~L} 2$ & $8 \mathrm{~L} 2$ \\
0 & 100 & $4 \mathrm{~L} 3$ & $8 \mathrm{~L} 3$ \\
\hline
\end{tabular}

Tabla 2. Efecto de diferentes sustratos (\% de turba/perlita en la mezcla) y tamaños de contenedor (4 L y 8 L) sobre longitud de pedúnculo, diámetro de capítulo y diámetro de pedúnculo, producción de flores y consumo de agua de un cultivo de gerbera en a) ciclo 1 (otoño: marzo-julio), y b) ciclo 2 (primavera: agosto-noviembre), en el primer año de producción

Table 2. Effect of different growing media (\% of peat/perlite in the mixture) and container size (4 and $8 \mathrm{~L}$ ) on peduncle lenght, capitulum diameter and peduncle diameter, flower production and water consumption of a gerbera cut flower crop in a) bloom 1 (auntumm: march-july), and b) bloom 2 (spring: august-november), at the first year of production

A)

\begin{tabular}{|c|c|c|c|c|c|c|c|c|c|c|}
\hline \multirow{2}{*}{$\begin{array}{c}\text { Tratamiento } \\
4 \mathrm{~L} 1\end{array}$} & \multirow{2}{*}{$\begin{array}{c}\begin{array}{c}\% \text { Turba / } \\
\text { Perlita }\end{array} \\
50 / 50\end{array}$} & \multirow{2}{*}{$\frac{\text { Cont. }}{4 \mathrm{~L}}$} & \multicolumn{2}{|c|}{$\begin{array}{l}\text { Largo de } \\
\text { tallo }(\mathrm{cm})\end{array}$} & \multicolumn{2}{|c|}{$\begin{array}{l}\text { Diámetro de } \\
\text { capítulo }(\mathrm{cm})\end{array}$} & \multicolumn{2}{|c|}{$\begin{array}{l}\text { Diámetro de } \\
\text { tallo }(\mathrm{mm})\end{array}$} & \multirow{2}{*}{ 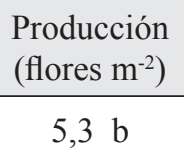 } & \multirow{2}{*}{$\begin{array}{c}\begin{array}{l}\text { Consumo de } \\
\text { agua }\left(\mathrm{L} \mathrm{m}^{-2}\right)\end{array} \\
23,5 \mathrm{~b}\end{array}$} \\
\hline & & & 42,16 & $\mathrm{c}$ & 9,53 & $\mathrm{~b}$ & 6,81 & $\mathrm{a}$ & & \\
\hline $4 \mathrm{~L} 2$ & $25 / 75$ & $4 \mathrm{~L}$ & 46,81 & $\mathrm{~b}$ & 10,39 & $\mathrm{a}$ & 6,68 & $\mathrm{a}$ & $9,2 \mathrm{~b}$ & $19,4 \mathrm{ab}$ \\
\hline $4 \mathrm{~L} 3$ & $0 / 100$ & $4 \mathrm{~L}$ & 48,83 & $\mathrm{~b}$ & 10,14 & $\mathrm{a}$ & 6,85 & $\mathrm{a}$ & $3,8 \mathrm{~b}$ & $19,7 \mathrm{ab}$ \\
\hline $8 \mathrm{~L} 1$ & $50 / 50$ & $8 \mathrm{~L}$ & 51,08 & $\mathrm{a}$ & 10,20 & $\mathrm{a}$ & 7,00 & $\mathrm{a}$ & $8,4 \mathrm{~b}$ & 14,4 a \\
\hline $8 \mathrm{~L} 2$ & $25 / 75$ & $8 \mathrm{~L}$ & 52,58 & $\mathrm{a}$ & 10,06 & $\mathrm{ab}$ & 6,96 & $\mathrm{a}$ & $8,4 \mathrm{~b}$ & $17,7 \mathrm{a}$ \\
\hline $8 \mathrm{~L} 3$ & $0 / 100$ & $8 \mathrm{~L}$ & 48,50 & $\mathrm{~b}$ & 9,56 & $\mathrm{~b}$ & 6,23 & $\mathrm{a}$ & 18,3 a & $19.9 \mathrm{ab}$ \\
\hline
\end{tabular}

B)

\begin{tabular}{ccccccccccc}
\hline Tratamiento & $\begin{array}{c}\text { \% Turba / } \\
\text { Perlita }\end{array}$ & Cont. & \multicolumn{2}{c}{$\begin{array}{c}\text { Largo de } \\
\text { tallo }(\mathrm{cm})\end{array}$} & $\begin{array}{c}\text { Diámetro de } \\
\text { capítulo }(\mathrm{cm})\end{array}$ & $\begin{array}{c}\text { Diámetro de } \\
\text { tallo }(\mathrm{mm})\end{array}$ & $\begin{array}{c}\text { Producción } \\
\left(\text { flores } \mathrm{m}^{-2}\right)\end{array}$ & $\begin{array}{c}\text { Consumo de } \\
\text { agua }\left(\mathrm{L} \mathrm{m}^{-2}\right)\end{array}$ \\
\hline 4L1 & $50 / 50$ & $4 \mathrm{~L}$ & 42,32 & $\mathrm{~b}$ & 10,18 & $\mathrm{a}$ & 6,54 & $\mathrm{a}$ & $15,3 \mathrm{~cd}$ & $61,9 \mathrm{~b}$ \\
4L2 & $25 / 75$ & $4 \mathrm{~L}$ & 46,57 & $\mathrm{ab}$ & 10,76 & $\mathrm{a}$ & 6,51 & $\mathrm{a}$ & $21,4 \mathrm{abc}$ & $61.3 \mathrm{~b}$ \\
4L3 & $0 / 100$ & $4 \mathrm{~L}$ & 47,99 & $\mathrm{a}$ & 10,49 & $\mathrm{a}$ & 5,92 & $\mathrm{ab}$ & $16,8 \mathrm{ab}$ & $58,2 \mathrm{~b}$ \\
8L1 & $50 / 50$ & $8 \mathrm{~L}$ & 48,54 & $\mathrm{a}$ & 10,50 & $\mathrm{a}$ & 6,15 & $\mathrm{ab}$ & $9,9 \mathrm{~d}$ & $41,8 \mathrm{a}$ \\
8L2 & $25 / 75$ & $8 \mathrm{~L}$ & 50,48 & $\mathrm{a}$ & 9,97 & $\mathrm{a}$ & 6,20 & $\mathrm{ab}$ & $21,4 \mathrm{abc}$ & $45,7 \mathrm{a}$ \\
8L3 & $0 / 100$ & $8 \mathrm{~L}$ & 48,37 & $\mathrm{a}$ & 9,91 & $\mathrm{a}$ & 5,71 & $\mathrm{~b}$ & $35,4 \mathrm{a}$ & $46,2 \mathrm{a}$ \\
\hline
\end{tabular}

Letras diferentes entre sustratos y contenedores indican diferencias significativas $(\mathrm{p} \leq 0.05)$. 
Tabla 3. Resumen de efectos de contenedor (c), sustrato (s) e interacción (c x s) sobre el rendimiento, la calidad de flor y consumo de agua en el ciclo 1 (otoño: marzo-julio) y ciclo 2 (primavera: agosto-noviembre) del primer año de producción Table 3. Summary of container (c), growing media (s) and interaction (c x s) effects on yield, flower quality and water consumption in a) bloom 1 (auntumm: march-july), and b) bloom 2 (spring: august-november), at the first year of production

\begin{tabular}{|c|c|c|c|c|}
\hline \multirow[b]{2}{*}{ variables de cultivo } & \multirow[b]{2}{*}{ Ciclo } & \multicolumn{2}{|c|}{ Efectos principales } & \multirow{2}{*}{$\begin{array}{l}\text { Interacción } \\
\mathrm{C} \times \mathrm{S}\end{array}$} \\
\hline & & Contenedor (C) & $\begin{array}{l}\text { Sustrato } \\
\text { (S) }\end{array}$ & \\
\hline \multirow{2}{*}{ Flores $\mathrm{m}^{-2}$} & 1 & $* *$ & $\mathrm{~ns}$ & $*$ \\
\hline & 2 & $*$ & $*$ & $* *$ \\
\hline \multirow{2}{*}{ Largo de tallo } & 1 & $* *$ & $*$ & $*$ \\
\hline & 2 & $* *$ & $\mathrm{~ns}$ & $\mathrm{~ns}$ \\
\hline \multirow{2}{*}{ Diámetro de tallo } & 1 & ns & $\mathrm{ns}$ & $\mathrm{ns}$ \\
\hline & 2 & $\mathrm{~ns}$ & $\mathrm{~ns}$ & $*$ \\
\hline \multirow{2}{*}{ Diámetro de capítulo } & 1 & $\mathrm{~ns}$ & $\mathrm{~ns}$ & $*$ \\
\hline & 2 & ns & $\mathrm{ns}$ & $\mathrm{ns}$ \\
\hline \multirow{2}{*}{ Consumo de agua } & 1 & $*$ & $\mathrm{~ns}$ & $*$ \\
\hline & 2 & $* *$ & $\mathrm{~ns}$ & ns \\
\hline
\end{tabular}

$\mathrm{ns}, * \mathrm{y} * *$ denotan no significativo o significativo a $\mathrm{p} \leq 0.05$ y $0.01 \%$ respectivamente.



Figura 1. Producción total de flores cortadas y consumo de agua por flor cosechada en un cultivo de gerbera en diferentes tamaños de contenedor $(4$ y $8 \mathrm{~L}$ ) y sustratos (\% de turba/perlita) desde marzo a noviembre de 2006. Fecha de plantación: Enero 2006. Letras diferentes indican diferencias significativas entre tratamientos para producción total (minúsculas) y consumo de agua por flor cosechada (mayúsculas). Barras oscuras: contenedores de $8 \mathrm{~L}$, barras claras: contenedores de $4 \mathrm{~L}$.

Figure 1. Total cut flower production and water consumption per harvested flower of a gerbera cut flower crop in different container size (4 and $8 \mathrm{~L}$ ) and growing media (peat/perlite \%) from March to November 2006. Planting date: January 2006. Different letters indicate significant differences among treatments for total production (small letters) and water consumption per flower harvested (capital letters). dark bars: $8 \mathrm{~L}$ containers, clear bars: 4 L containers. 